\title{
$K$-theory, flat bundles and the Borel classes
}

\author{
by
}

\author{
Bjørn J a hren (Oslo)
}

\begin{abstract}
Using Hausmann and Vogel's homology sphere bundle interpretation of algebraic $K$-theory, we construct $K$-theory invariants by a theory of characteristic classes for flat bundles. It is shown that the Borel classes are detected this way, as well as the rational $K$-theory of integer group rings of finite groups.
\end{abstract}

1. Introduction. One of the basic problems with algebraic $K$-theory is the lack of a good interpretation (geometric, algebraic or otherwise) of the elements in the higher $K$-groups. This makes calculations harder, but perhaps more importantly, it complicates applications, since even in the cases where one can do calculations, the connection to the application is often so indirect that it is hard to translate back.

To give just one example: Borel's calculations show that $K_{4 k+1}(\mathbb{Z}) \otimes \mathbb{Q}$ $\approx \mathbb{Q}$ for $k \geq 1$. One consequence is that some homotopy groups of diffeomorphism groups of large discs (fixing the boundary) have rank one. But to construct explicit generators from these calculations seems to be very difficult!

If $A$ is a ring, $K_{n}(A)$ is defined as $\pi_{n}\left(B G L(A)^{+}\right)$, i.e. by mapping simple spaces (spheres) into complicated and poorly understood spaces $\left(B G L(A)^{+}\right)$. In this paper we discuss an alternative (but equivalent) definition, where we replace the spheres by more complicated manifolds, but map into spaces that are much better understood (at least from a geometric point of view) - namely the classifying spaces $B G L(A)$. Then the elements have geometric interpretation as (flat) bundles, and one can try to study invariants for these.

The idea to construct geometric invariants for $K$-theory elements from a flat bundle representation was, I believe, first used by Karoubi. He used a representation by bundles over $K(\pi, 1)$-complexes and developed a theory of simplicial connections and characteristic classes (see [9]). Here, however,

1991 Mathematics Subject Classification: Primary 19D50; Secondary 57R20. 
we shall use an interpretation given by Hausmann and Vogel in [5], using bundles over homology spheres which are actual manifolds. In fact, we shall use a differentiable adaptation of Hausmann and Vogel's theory (which works in the PL category). This has the advantage that we can apply directly a differential geometric theory of characteristic classes.

It should be noted, however, that Karoubi's bundle interpretation is slightly more general, since it works in the same manner in all degrees, whereas that of Hausmann and Vogel only works in degrees higher than 4.

Hausmann and Vogel's theory is recalled in Section 2, together with a discussion of the differentiable case ([5] works in the PL category).

In Section 3 we discuss a theory of characteristic classes for flat bundles (essentially due to Kamber and Tondeur), which we apply in Section 4 to give invariants in the cases $A=\mathbb{R}, \mathbb{C}$ or $\mathbb{H}$. In Section 5 we compare with Borel's calculations, and prove that these can be formulated in terms of the invariants from Section 4 . Hence these invariants are highly nontrivial, since they detect the ranks of $K$-groups of algebraic integers. In fact, they also give a complete set of rational invariants in the case of group rings of finite groups. This case is discussed briefly in Section 6. (For more details, see [6].)

2. Homology sphere bordism and $K$-theory. The starting point is the "geometric" interpretation of the homotopy of the plus-construction provided by Hausmann and Vogel in [5] (see also [4]):

Let $X$ be a pointed space. We say that two pointed maps $f_{i}: M_{i}^{n} \rightarrow X$, $i=1,2$, from closed, oriented $n$-manifolds $M_{1}$ and $M_{2}$ to $X$ are homology cobordant if there exists an oriented, pointed cobordism $F: W \rightarrow X$ between $f_{1}$ and $f_{2}$ such that

$$
H_{*}\left(W, M_{1} ; \mathbb{Z}\right) \approx H_{*}\left(W, M_{2} ; \mathbb{Z}\right) \approx 0 .
$$

(The cobordism is pointed if there is an arc in $W$ joining the basepoints in $M_{1}$ and $M_{2}$ and which is mapped to the basepoint in $X$ by $F$.)

Let $\Omega_{n}^{\mathrm{HS}}(X)$ be the set of homology cobordism classes of maps $f: M^{n}$ $\rightarrow X$ where $M$ is an oriented homology sphere. Oriented connected sum gives $\Omega_{n}^{\mathrm{HS}}(X)$ the structure of an abelian group.

This makes sense in the topological, PL or differentiable category, and if we want to emphasize which category we are in, we write $\Omega_{n}^{\mathrm{HS}}(X)_{\mathcal{C A T}}$, where $\mathcal{C} \mathcal{A} \mathcal{T}=$ TOP, PL, or DIFF.

For any space $Y$, we let $Y^{+}$denote the result of the plus-construction on $Y$ with respect to the maximal locally perfect subgroup $L P \pi_{1}(Y)$ of $\pi_{1}(Y)$ ([5]). If $W$ is a cobordism between homology $n$-spheres $M_{1}$ and $M_{2}$, then $\left(W^{+} ; M_{1}^{+}, M_{2}^{+}\right) \simeq\left(\mathrm{S}^{n} \times I ; \partial\left(\mathrm{S}^{n} \times I\right)\right)$, so $(f: M \rightarrow X) \mapsto$ $\left(f^{+}: M^{+} \rightarrow X^{+}\right)$defines a map $\Omega_{n}^{\mathrm{HS}}(X) \rightarrow \pi_{n}\left(X^{+}\right)$which is easily seen to be a homomorphism. 
In the PL category Hausmann and Vogel prove ([5]):

THEOREM 2.1. (a) $\Omega_{n}^{\mathrm{HS}}(X)_{\mathrm{PL}} \rightarrow \pi_{n}\left(X^{+}\right)$is an isomorphism if $n \geq 5$.

(b) There are exact sequences

$$
\begin{gathered}
0 \rightarrow \Omega_{4}^{\mathrm{HS}}(X)_{\mathrm{PL}} \rightarrow \pi_{4}\left(X^{+}\right) \rightarrow \Omega_{3}^{\mathrm{HS}}(\mathcal{F})_{\mathrm{PL}} \rightarrow \Omega_{3}^{\mathrm{HS}}(X)_{\mathrm{PL}} \rightarrow \pi_{3}\left(X^{+}\right) \rightarrow 0, \\
0 \rightarrow \Omega_{2}^{\mathrm{HS}}(X)_{\mathrm{PL}} \rightarrow \pi_{2}\left(X^{+}\right) \rightarrow H_{2}\left(L P \pi_{1}(X) ; \mathbb{Z}\right) \rightarrow 0,
\end{gathered}
$$

where $\mathcal{F}$ is the homotopy fiber of the plus-map $X \rightarrow X^{+}$.

REMARK 2.2. In (b) there is no difference between the PL and differentiable categories, so we get the same statement for $\Omega_{n}^{\mathrm{HS}}(X)_{\mathrm{DIFF}}$.

In order to get the necessary modifications for the differentiable category (for $n \geq 5$ ), we investigate the relation between $\Omega_{n}^{\mathrm{HS}}(X)_{\mathrm{PL}}$ and $\Omega_{n}^{\mathrm{HS}}(X)_{\mathrm{DIFF}}$.

TheOrem 2.3. For every $n$ there is a functorial split exact sequence

$$
0 \rightarrow \Gamma_{n} \stackrel{\iota}{\rightarrow} \Omega_{n}^{\mathrm{HS}}(X)_{\mathrm{DIFF}} \stackrel{\varrho}{\rightarrow} \Omega_{n}^{\mathrm{HS}}(X)_{\mathrm{PL}} \rightarrow 0
$$

where $\Gamma_{n}$ is the group of concordance classes of differentiable structures on the $n$-sphere.

Proof. For $n \leq 5$ there is no difference between the differentiable and PL case, and $\Gamma_{n}=0$. Hence we assume $n \geq 6$. (The argument actually works for $n \geq 5$.)

By [10], every PL homology $n$-sphere $M, n \neq 3$, is the boundary of a contractible manifold, which by smoothing theory has a (unique) differentiable structure. Therefore $M$ has a differentiable structure, so it follows that $\Omega_{n}^{\mathrm{HS}}(X)_{\mathrm{DIFF}} \rightarrow \Omega_{n}^{\mathrm{HS}}(X)_{\mathrm{PL}}$ is surjective.

The map $\iota: \Gamma_{n} \rightarrow \Omega_{n}^{\mathrm{HS}}(X)_{\text {DIFF }}$ takes a homotopy sphere $\Sigma$ to the constant map $\Sigma \rightarrow X$. This is clearly a homomorphism, since addition in $\Gamma_{n}$ is also given by connected sum.

$\iota$ is injective: Let $W$ be a homology cobordism between $\Sigma^{n}$ and (standard) $S^{n}$. Then the plus-construction on $W$ can be realized by surgeries on one- and two-handles in the interior. Thus we obtain an $h$-cobordism between $\Sigma^{n}$ and $\mathrm{S}^{n}$, which is trivial since $n \geq 5$.

It is obvious that $\varrho \circ \iota=0$, so it remains to prove that ker $\varrho \subseteq \iota\left(\Gamma_{n}\right)$.

Let $f: M \rightarrow X$ represent an element $\alpha \in$ ker $\varrho$. Then $f$ can be extended to a map $F: W \rightarrow X$, where $\partial W=M$ and $H_{*}(W ; \mathbb{Z}) \approx H_{*}($ point; $\mathbb{Z})$. Deleting a small disk in $W$, we obtain a (PL) homology cobordism $F^{\prime}$ : $W^{\prime} \rightarrow X$ between $f$ and the constant map $\mathrm{S}^{n} \rightarrow X$. By smoothing theory, the differentiable structure on $M$ can be extended (in fact uniquely) to a differentiable structure on $W^{\prime}$. Restricting to the other end, we get a differentiable structure $\gamma$ on $\mathrm{S}^{n}$. Then $\alpha=\iota(\gamma)$. 
Functoriality of the sequence is obvious. But then the splitting is obtained by mapping the sequence for $X$ to the sequence for a point, since $\Omega_{n}^{\mathrm{HS}}(*)_{\mathrm{PL}}$ is trivial by the above mentioned result of Kervaire [10].

Corollary 2.4. For $n \geq 5$ there is a split exact sequence

$$
0 \rightarrow \Gamma_{n} \rightarrow \Omega_{n}^{\mathrm{HS}}(X)_{\mathrm{DIFF}} \rightarrow \pi_{n}\left(X^{+}\right) \rightarrow 0 .
$$

We now specialize to the case we are interested in, namely $X=B G L(A)$ for a ring $A$. As observed in [5], $E(A)$ (the subgroup of $G L(A)$ generated by elementary matrices) is locally perfect, so $\pi_{n}\left(X^{+}\right) \approx K_{n}(A)$. From now on we only consider the differentiable homology bordism groups, so we simplify the notation by writing $\Omega_{n}^{\mathrm{HS}}(X)=\Omega_{n}^{\mathrm{HS}}(X)_{\mathrm{DIFF}}$.

From the results above, we then have exact sequences

$$
\begin{aligned}
0 \rightarrow \Gamma_{n} \rightarrow \Omega_{n}^{\mathrm{HS}}(B G L(A)) \rightarrow & K_{n}(A) \rightarrow 0 \quad(n \geq 5), \quad \text { and } \\
0 \rightarrow \Omega_{4}^{\mathrm{HS}}(B G L(A)) \rightarrow K_{4}(A) \rightarrow \Omega_{3}^{\mathrm{HS}}(\mathcal{F}) & \rightarrow \Omega_{3}^{\mathrm{HS}}(B G L(A)) \rightarrow K_{3}(A) \rightarrow 0
\end{aligned}
$$

$\left(\mathcal{F}\right.$ is the homotopy fiber of $\left.B G L(A) \rightarrow B G L(A)^{+}\right)$.

But generators of $\Omega_{n}^{\mathrm{HS}}(B G L(A))$ can also be thought of as classifying maps for principal $G L(A)$-bundles (or $G L_{k}(A)$-bundles for large $k$ )actually covering spaces - over homology $n$-spheres. Hence we get the following interpretation of $K_{n}(A), n \geq 5$ :

$K_{n}(A) \approx$ \{principal $G L_{k}(A)$-bundles over smooth homology $n$-spheres $\} / \sim$ where the equivalence relation $\sim$ is generated by isomorphism, homology cobordism, stabilization (in $k$ ), and change of differentiable structure.

REMARKS 2.7. (1) The cases $n=3$ and $n=4$ are more mysterious. For $n=3$ we obviously get a similar interpretation, but with more relations. For $n=4$ we may not get all of $K_{4}(A)$. It is conceivable that the exact sequence (2.6) splits into an isomorphism for $n=4$ and a short exact sequence. However, $\Omega_{3}^{\mathrm{HS}}(\mathcal{F})$ is definitely not trivial, since it contains $\Omega_{3}^{\mathrm{HS}}$ (point) $=$ $\theta_{3}$ - the group of homology 3 -spheres - as a direct summand, and this is now known to be big. One might try to use $\Omega_{3}^{\mathrm{HS}}(\mathcal{F})_{\text {TOP }}$ instead, and an optimistic conjecture would be that this is trivial.

For $n=1$ and $n=2$, however, it is easy to see what happens.

Since $K_{1}(A)=H_{1}(B G L(A))$, we can describe $K_{1}$ by the same generators, but with arbitrary cobordisms as relations.

Similarly, since $K_{2}(A)=H_{2}(E(A))$, where $E(A)$ is the subgroup of $G L(A)$ generated by elementary matrices, we can describe $K_{2}$ as a cobordism group of $E(A)$-bundles, but this time over arbitrary 2-manifolds. The construction of the corresponding element in $\pi_{2} B G L(A)^{+}$in this case goes as before, but we have to kill all of $\pi_{1} M$, even if it is not (locally) perfect. 
(2) For $n \geq 2$ we can replace $G L(A)$ by $E(A)$ or $S L(A)$ when $A$ is commutative. In the following we shall sometimes do this without further comment.

ExAmple 2.8. (i) In [7], Jones and Westbury construct examples of flat bundles over 3-dimensional homology spheres, representing elements of $K_{3}(\mathbb{C})$. For instance, they show that hyperbolic homology spheres naturally give rise to elements of infinite order, and every element of finite order can be represented by flat bundles over Seifert homology spheres of type $\Sigma(p, q, r)$. Such bundles are determined by a complex representation of the fundamental group, and observing that some of these representations are, in fact, defined over integers in certain number fields, one also can represent elements of $K$-theory of these rings. One particularly nice example is the Poincaré sphere, whose fundamental group is a subgroup of $S U_{2}$. This subgroup may be defined over the cyclotomic integers $\mathbb{Z}\left[\zeta_{5}\right]$, and Jones and Westbury show that the resulting element in $K_{3}\left(\mathbb{Z}\left[\zeta_{5}\right]\right)$ generates the torsion subgroup (which is isomorphic to $\mathbb{Z} / 120$ ).

(ii) Another interesting case is $X=B S_{\infty}$, the classifying space of the infinite symmetric group. Then the Barratt-Priddy-Quillen theorem says that $\pi_{n}\left(X^{+}\right) \approx \pi_{n}^{\mathrm{S}}$, the stable homotopy groups of spheres, for $n>0$. Therefore these also have homology bordism interpretation.

The natural inclusion of $S_{k}$ in $G L_{k}(\mathbb{Z})$ as the permutation matrices induces a diagram

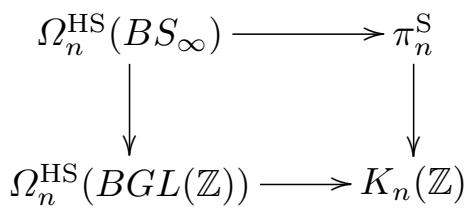

where the right hand vertical map is the standard homomorphism.

For $n=3$ the horizontal homomorphisms are still surjective, and it would be interesting to have nice descriptions of homology 3 -spheres and bundles representing the generators of $\pi_{3}^{\mathrm{S}}$ and $K_{3}(\mathbb{Z})$.

With the bundle interpretation of (elements of) $K$-theory groups, it is natural to look for invariants in terms of characteristic classes. Our aim is to use a simple differential geometric approach related to the Chern-Weil theory, but measuring the incompatibility of the discrete and the metric structure on a flat bundle. We shall do this first in the case of $A$ equal to the real, complex, or quaternionic numbers. For other rings one can use this case to get invariants as follows:

Suppose we have a representation $A \rightarrow M_{q}(F)(=q \times q$-matrices with entries in $F$ ), where $F=\mathbb{R}, \mathbb{C}$ or $\mathbb{H}$. Then we have an induced map $K_{*}(A) \rightarrow$ 
$K_{*}\left(M_{q}(F)\right) \approx K_{*}(F)$ in $K$-theory. In the bundle interpretation, this takes a principal $G L_{k}(A)$-bundle over $M$ to the associated $G L_{k q}(F)$-bundle over $M$.

$G L_{k}(F)$-bundles can be thought of as (principal bundles associated with) flat F-vector bundles, so what we shall do in the next two sections is first to study a general theory of characteristic classes for flat bundles, and then apply it to bundles over homology spheres.

3. Characteristic classes for flat bundles. In this section we sketch the constructions of characteristic classes for foliated bundles in [8], Ch. 3, and apply it to our situation. For details, see [8].

Suppose that we have a differentiable, principal $G$-bundle $\pi: P \rightarrow M$ over a manifold $M$, where $G$ is a Lie group. Then $P$ has a free right $G$-action $\mu: P \times G \rightarrow P$ with quotient $M$. We write $\mu(p, g)=L_{p}(g)=R_{g}(p)$ - the same notation as for left and right multiplication in $G$.

Let $\mathfrak{g}$ be the Lie algebra of $G$. If $p \in P$, then the differential of $L_{p}$ at the unit $e \in G$ is a monomorphism $L_{p *}: \mathfrak{g} \rightarrow T_{p} P$. Any $x \in \mathfrak{g}$ then determines a vector field $\bar{x}$ on $P$ by $\bar{x}(p)=L_{p *}(x)$.

In Cartan's formalism, a connection in the principal bundle is then a splitting $\bar{\omega}_{p}: T_{p} P \rightarrow \mathfrak{g}$ of $L_{p *}$ for every $p \in P$ such that

(1) $\bar{\omega}_{p}$ depends differentiably on $p$ in the sense that they collect to a differentiable map $T P \rightarrow \mathfrak{g}$.

(2) $R_{g^{-1}} \bar{\omega}=\operatorname{Ad}_{g} \bar{\omega}$ ( $\bar{\omega}$ is $G$-invariant).

$\bar{\omega}$ is a 1 -form on $P$ with values in the Lie algebra $\mathfrak{g}$, but we shall rather think of the connection as the homomorphism

$$
\omega: \mathfrak{g}^{*} \rightarrow \Omega^{1}(P)
$$

defined by $\phi \mapsto \phi \circ \bar{\omega}$.

$\mathfrak{g}^{*}$ and $\Omega^{1}(P)$ are both the degree one parts of natural differential graded algebras (DGAs): the Koszul complex $\left(\Lambda^{*}(\mathfrak{g}), d_{\Lambda}\right)$ which computes the Lie algebra cohomology of $\mathfrak{g}$, and the de Rham complex $\left(\Omega^{*}(P), d_{\Omega}\right)$. Clearly, $\omega$ extends to a map of graded algebras

$$
\omega: \Lambda^{*}(\mathfrak{g}) \rightarrow \Omega^{*}(P),
$$

but this does not in general commute with differentials:

Consider the following diagram:

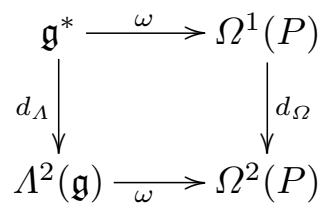

A calculation shows that: 
(1) If this diagram commutes, then $\omega: \Lambda^{*}(\mathfrak{g}) \rightarrow \Omega^{*}(P)$ commutes with differentials.

(2) The map $d_{\Omega} \circ \omega-\omega \circ d_{\Lambda}: \mathfrak{g}^{*} \rightarrow \Omega^{2}(P)$ can be identified with the curvature $K \in \Omega^{2}(P ; \mathfrak{g})$ of the connection.

It follows that $\omega$ extends to a map of DGAs precisely if the connection is flat!

So, if the connection is flat, we obtain a homomorphism from the Lie algebra cohomology $H_{\mathrm{LIE}}^{*}(\mathfrak{g})$ of $\mathfrak{g}$ to the de Rham cohomology $H_{\mathrm{DR}}^{*}(P)$ of $P$. However, this is not yet quite what we want.

By a flat bundle we shall mean a bundle with a flat connection. This means that the structure group can be considered with the discrete topology. Conversely, a bundle with discrete structure group has a unique flat connection.

Let now $H$ be a closed, connected subgroup of $G$, and consider the projection $\pi_{H}: P \rightarrow P / H$. This induces a monomorphism $\pi_{H}^{*}: \Omega^{*}(P / H) \rightarrow$ $\Omega^{*}(P)$ with image equal to the set of forms on $P$ which are $H$-invariant and which vanish on all vector fields of the form $\bar{x}$ for $x$ in the Lie algebra $\mathfrak{h}$ of $H$. This subspace can be characterized completely in terms of the infinitesimal action of $H$ as

$$
\operatorname{im}\left(\pi_{H}^{*}\right)=\left\{\tau \in \Omega^{*}(P) \mid i_{\bar{x}} \tau=0, \operatorname{ad}_{\bar{x}} \tau=0, \text { for all } x \in \mathfrak{h}\right\} .
$$

The right hand side of $(3.1)$ will be denoted by $\Omega^{*}(P)_{\mathfrak{h}}$. We have similar actions of $\mathfrak{h}$ on $\Lambda^{*}(\mathfrak{g})$, so we can define

$$
\Lambda^{*}(\mathfrak{g})_{\mathfrak{h}}=\left\{\tau \in \Lambda^{*}(\mathfrak{g}) \mid i_{x} \tau=0, \operatorname{ad}_{x} \tau=0, \text { for all } x \in \mathfrak{h}\right\} .
$$

It turns out that $\Lambda^{*}(\mathfrak{g})_{\mathfrak{h}} \subset \Lambda^{*}(\mathfrak{g})$ is again a DGA, and $\omega$ induces a restriction

$$
\omega_{\mathfrak{h}}: \Lambda^{*}(\mathfrak{g})_{\mathfrak{h}} \rightarrow \Omega^{*}(P)_{\mathfrak{h}} .
$$

The homology of $\Lambda^{*}(\mathfrak{g})_{\mathfrak{h}}$ is the relative Lie algebra cohomology and is denoted by $H_{\text {LIE }}^{*}(\mathfrak{g} ; \mathfrak{h})$. Hence we have obtained a homomorphism

$$
H_{\mathrm{LIE}}^{*}(\mathfrak{g} ; \mathfrak{h}) \rightarrow H_{\mathrm{DR}}^{*}(P / H) .
$$

Assume now that the structure group of the original bundle $P \rightarrow M$ can be reduced to $H$, but not necessarily as flat bundles. Then there is a section $M \rightarrow P / H$ of the bundle $P / H \rightarrow M$, and we get a composed homomorphism

$$
\alpha=\alpha_{P}: H_{\mathrm{LIE}}^{*}(\mathfrak{g} ; \mathfrak{h}) \rightarrow H_{\mathrm{DR}}^{*}(P / H) \rightarrow H_{\mathrm{DR}}^{*}(M) .
$$

REMARKS 3.2. (1) Let $P(H)$ be the principal $H$-bundle of the reduction. Then $P(H)$ is a sub- $H$-bundle of $P$. If the connection can also be reduced 
to $H$, we have a commutative diagram

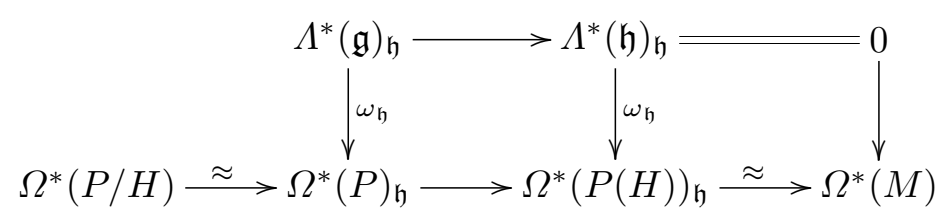

The bottom horizontal composition is precisely the splitting of $\Omega^{*}(M) \rightarrow$ $\Omega^{*}(P / H)$ given by reduction of the structure group, so $\alpha$ is trivial if the bundle has a flat reduction. Hence $\alpha \in \operatorname{Hom}\left(H_{\mathrm{LIE}}^{*}(\mathfrak{g} ; \mathfrak{h}), H_{\mathrm{DR}}^{*}(M)\right)$ is an obstruction to flat reduction of the structure group.

(2) The obvious application of $\alpha$ would then be to the investigation of flat bundles with (nonflat) reductions on some fixed manifold $M$. Here, however, we shall fix the pair $(G, H)$ and vary $M$ within its homology type.

For the applications in Section 4, we need the following

Proposition 3.4. Let $W$ be a homology cobordism between two manifolds $M_{1}$ and $M_{2}$, and assume that $Q \rightarrow W$ is a flat, principal G-bundle with an $H$-reduction $\sigma: W \rightarrow Q / H$. Restriction to $M_{i}$ then gives principal $G$-bundles $P_{i} \rightarrow M_{i}$ with $H$-reductions $\sigma_{i}=\sigma \mid M_{i}: M_{i} \rightarrow P_{i} / H$. Then the following diagram commutes:

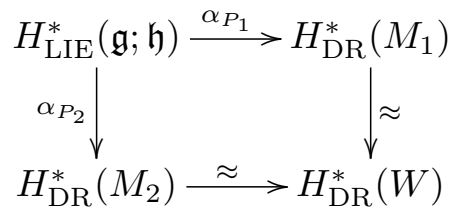

and the two compositions are equal to $\alpha_{Q}$.

Proof. This follows immediately from the commutative diagram

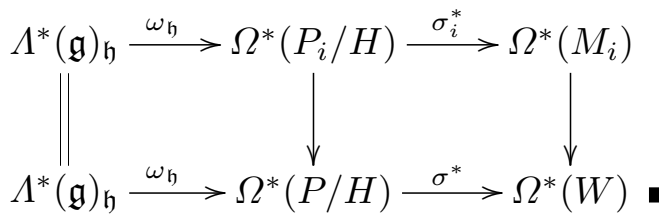

4. Application to $K$-theory. We now specialize to the case where $M$ is an oriented homology $n$-sphere and $G=G L_{q}^{0}(L)$, where $L$ is a finitedimensional, semisimple algebra over $\mathbb{R}$ and $G L_{q}^{0}(L)$ is the identity component of $G L_{q}(L)$. ( $L$ has a vector space structure such that the algebraic structure on $L$ is smooth and then $G L_{q}(L)$ also has the structure of a Lie group.) Then we can always reduce the structure group to the maximal compact subgroup $K=K_{q}$. In fact, $P / K \simeq M$, so $H_{\mathrm{DR}}^{*}(P / K) \rightarrow H_{\mathrm{DR}}^{*}(M)$ is 
uniquely determined (inverse of an isomorphism) and hence $\alpha$ is independent of the choice of reduction.

$M$ has interesting homology only in dimension $n$, and $H_{\mathrm{DR}}^{n}(M) \approx \mathbb{R}$, by an isomorphism which is uniquely determined by the orientation of $M$. Therefore we can think of $\alpha$ as an element in $\left(H_{\mathrm{LIE}}^{n}(\mathfrak{g} ; \mathfrak{k})\right)^{*}$. In fact, using the product structure, we see that $\alpha$ must vanish on all decomposables, i.e. on products of at least two elements of positive degree. Hence we can consider $\alpha$ an element of $\left(H_{\mathrm{LIE}}^{n}\left(\mathfrak{g} l_{q}(L), \mathfrak{k}_{q}\right) / \mathcal{D}\right)^{*}$, where $\mathcal{D}$ is the submodule generated by decomposables.

It follows from Proposition 3.4 that this construction induces a map

$$
\beta: \Omega_{n}^{\mathrm{HS}}\left(B G L_{q}^{0}(L)\right) \rightarrow\left(H_{\mathrm{LIE}}^{n}\left(\mathfrak{g l}_{q}(L), \mathfrak{k}_{q}\right) / \mathcal{D}\right)^{*},
$$

and this clearly commutes with stabilization in $q$. Since $\Gamma_{n}$ is finite, we then use (2.5) to get

THEOREM 4.1. $\beta$ induces a homomorphism

$$
B=B_{L}: K_{n}(L) \rightarrow \underset{q}{\lim }\left(H_{\mathrm{LIE}}^{n}\left(\mathfrak{g l}_{q}(L), \mathfrak{k}_{q}\right) / \mathcal{D}\right)^{*} .
$$

Pro of. It remains to prove that $\beta$ is additive. Suppose that $P \rightarrow M$ is the sum of $P_{1} \rightarrow M_{1}$ and $P_{2} \rightarrow M_{2}$, where $M=M_{1} \# M_{2}=M_{1}^{0} \cup_{n-1} M_{2}^{0}$, $M_{i}^{0}=M_{i}-\operatorname{int} D_{i}, D_{i}$ a small, open disc around the basepoint. Let further $P_{i}^{0}=P_{i} \mid M_{i}^{0}$.

Then we have a diagram of bundle maps

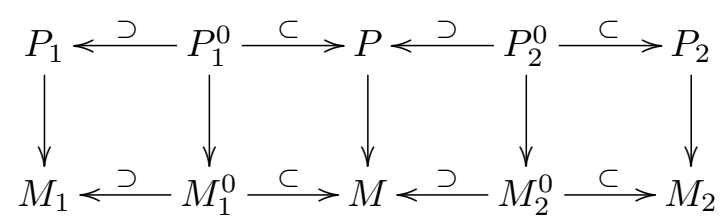

We may assume that the reductions to the maximal compact subgroup are compatible, such that the diagram

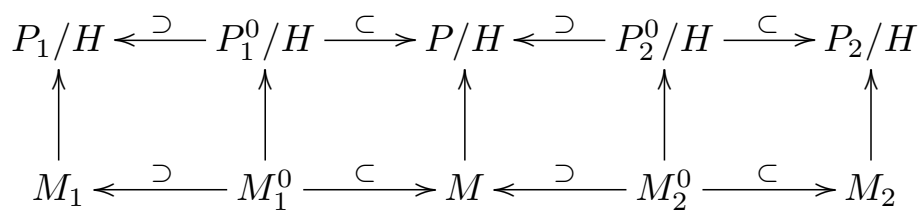

also commutes, where the vertical maps are sections of the induced bundle maps. Moreover, without loss of generality we may assume that the reductions are flat over $D_{i}$, resp. a small product neighborhood $\mathcal{U}$ of $n-1=$ $M_{1}^{0} \cap M_{2}^{0} \subset M$. 
Let $\varrho_{1}, \varrho_{2}$ be maps $M \rightarrow M_{i}$ such that $\varrho_{i}$ is the identity on $M_{i}^{0}$ and constant outside $M_{i} \cup \mathcal{U}$. Consider now the diagram

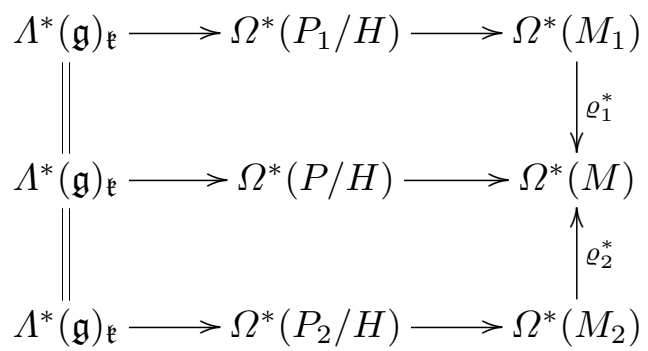

The three horizontal compositions induce $\alpha$ for the three bundles-let us denote them by $a_{P}, a_{P_{1}}$ and $a_{P_{2}}$. The diagram does not commute, but for every $\phi \in \Lambda^{*}(\mathfrak{g})_{\mathfrak{k}}$ we have

$$
a_{P}(\phi)_{x}=a_{P_{i}}(\phi)_{x} \quad \text { if } x \in M_{i}^{0}
$$

and since for every $\tau \in \Omega^{*}\left(M_{i}\right), \varrho_{i}^{*}(\tau)=0$ outside $M_{i} \cup \mathcal{U}$, we have

$$
a_{P}(\phi)_{x}=\varrho_{1}^{*} a_{P_{1}}(\phi)_{x}+\varrho_{2}^{*} a_{P_{2}}(\phi)_{x} \quad \text { for } x \in M-\mathcal{U} .
$$

But since the reductions can be assumed to be flat in $x$ and $\varrho_{i}(x)$ for $x \in \mathcal{U}$, it follows by inspection of diagram (3.3) that both sides of (4.3) are zero in $\mathcal{U}$.

Since $\varrho_{i}$ are degree one maps and hence canonically identify $H_{\mathrm{DR}}^{*}\left(M_{i}\right)$ and $H_{\mathrm{DR}}^{*}(M)$, the assertion follows.

The most important examples are $L=\mathbb{R}, \mathbb{C}$ or $\mathbb{H}$. Then

$$
(G, K)=\left(G L_{q}^{0}(\mathbb{R}), S O_{q}\right),\left(G L_{q}(\mathbb{C}), U_{q}\right) \text { or }\left(G L_{q}(\mathbb{H}), S p_{q}\right), \text { resp. }
$$

Define $P_{n}(F), F=\mathbb{R}, \mathbb{C}$ or $\mathbb{H}$, by

$$
P_{n}(F)= \begin{cases}\mathbb{R} & \text { if } n \equiv 1(\bmod 4), \\ \mathbb{R} & \text { if } n \equiv 1(\bmod 2) \text { and } F=\mathbb{C}, \\ 0 & \text { otherwise }\end{cases}
$$

Then we have the following calculations:

Proposition 4.2. Let $\mathfrak{k}_{q}(F)$ be the maximal compact Lie subalgebra of $\mathfrak{g l}_{q}(F)$. Then

$$
\left(H_{\mathrm{LIE}}^{n}\left(\mathfrak{g} l_{q}(F), \mathfrak{k}_{q}(F)\right) / \mathcal{D}\right)^{*} \approx P_{n}(F) \quad \text { for } q \gg n .
$$

Moreover, the isomorphisms can be chosen to be compatible with stabilization in $q$.

Pr o of. We sketch the idea of the proof of this well known fact. Each of the pairs of Lie algebras has a compact dual with the same cohomology. But this is the same as the de Rham cohomology of the corresponding homogeneous spaces. In the three cases $\mathbb{R}, \mathbb{C}$ and $\mathbb{H}$ we get $H_{\mathrm{DR}}^{*}(U / S O), H_{\mathrm{DR}}^{*}(U)$ 
and $H_{\mathrm{DR}}^{*}(U / S p)$ resp., stably. The duals of these cohomology rings $\bmod \mathcal{D}$ are the modules generated by primitive elements in real homology, which are isomorphic to the homotopy groups tensored by $\mathbb{R}$. But these are known from Bott periodicity computations.

Corollary 4.3. $\beta$ induces a homomorphism $B_{F}: K_{n}(F) \rightarrow P_{n}(F)$ for $n \geq 5$.

If, more generally, $L$ is a finite-dimensional, semisimple $\mathbb{R}$-algebra, we know from the structure theory that it is isomorphic to a product $L \approx$ $\prod_{i} M_{d_{i}}\left(F_{i}\right)$ of matrix algebras over $F_{i}=\mathbb{R}, \mathbb{C}$ or $\mathbb{H}$. Therefore, by Morita equivalence, $K_{n}(L) \approx \prod_{i} K_{n}\left(F_{i}\right)$, and hence we get homomorphisms

$$
B_{L}: K_{n}(L) \rightarrow \prod_{i} P_{n}\left(F_{i}\right)
$$

In Sections 5 and 6, we show that these homomorphisms are closely related to the Borel regulator maps, and hence highly nontrivial.

5. Comparison with Borel's computations. Let $k$ be a number field of degree $d$, and let $A_{k}$ be its ring of integers. Then $k$ has $r_{1}$ real and $r_{2}$ complex Archimedian valuations, where $d=r_{1}+2 r_{2}$.

Each such valuation $v$ determines an embedding $i_{v}$ of $k$ into the completion $k_{v}$, uniquely if $k_{v}=\mathbb{R}$ and a conjugate pair of embeddings if $k_{v}=\mathbb{C}$. Choosing one embedding for each conjugate pair, we get a product embedding of $k$ into the ring

$$
\widehat{k}=\prod_{v} k_{v}=\mathbb{R}^{r_{1}} \times \mathbb{C}^{r_{2}} .
$$

The important fact is now that $A_{k}$ is a lattice in $\widehat{k}$. In fact, we can think of $\widehat{k}$ as the decomposition of $A_{k} \otimes_{\mathbb{Z}} \mathbb{R}$ into simple algebras.

Now set $L=\widehat{k}$ in Theorem 4.1. Then $B_{L}$ in (4.4) reduces to the product of all the homomorphisms $B_{k_{v}}$ from Corollary 4.3. Hence we have homomorphisms

$$
\begin{aligned}
K_{n}\left(A_{k}\right) \rightarrow K_{n}(k) \rightarrow K_{n}(\mathbb{R})^{r_{1}} \times K_{n}(\mathbb{C})^{r_{2}} \\
\stackrel{B}{\rightarrow} \begin{cases}\mathbb{R}^{r_{1}+r_{2}} & \text { if } n \equiv 1(\bmod 4), \\
\mathbb{R}^{r_{2}} & \text { if } n \equiv 3(\bmod 4) .\end{cases}
\end{aligned}
$$

Call this composed homomorphism $B_{A}$. Recall that in [2], Borel computed the rank of $K_{n}\left(A_{k}\right)$ to be exactly the exponents on the right hand side for $n$ odd $>1$ (and 0 for $n$ even). We shall show that Borel's result can be formulated as follows:

Theorem 5.2. After tensoring $K_{n}\left(A_{k}\right)$ with $\mathbb{R}, B_{A}$ becomes an isomorphism. 
In other words, except for torsion, we detect all of $K_{n}\left(A_{k}\right)$ using the homomorphisms $\beta_{F}$ of Corollary 4.3 for all embeddings $A_{k} \rightarrow \mathbb{R}$ and $\mathbb{C}$.

REMARK. We really should not write the right hand side in (5.1) as powers of $\mathbb{R}$, since this involves choosing a basis for each $P_{n}\left(k_{v}\right)$. One way to do this is to use elements corresponding to integral generators of the homotopy of the homogeneous spaces giving rise to the computations sketched in Proposition 4.2. The comparison of the image of $B_{A}$ to the resulting basis of $\prod P_{n}\left(k_{v}\right)$ involves very interesting number theory, and gives rise to the so-called higher regulators ([11], [3]).

Proof of Theorem 5.2. Borel's computations use the fact that all vector spaces involved are finite-dimensional, and the isomorphisms

$$
K_{n}\left(A_{k}\right) \otimes \mathbb{Q} \approx \operatorname{Prim} H_{n}\left(S L\left(A_{k}\right) ; \mathbb{Q}\right) \approx\left(H^{n}\left(S L\left(A_{k}\right) ; \mathbb{Q}\right) / \mathcal{D}\right)^{*} \quad(n>1),
$$

to reduce the problem to that of computing $H^{*}\left(S L\left(A_{k}\right) ; \mathbb{Q}\right)$, or rather $H^{*}\left(S L\left(A_{k}\right) ; \mathbb{R}\right)$. (As above, $\mathcal{D}$ is the module generated by products.)

To do this, he constructs an algebra homomorphism

$$
j: I_{G_{q}} \rightarrow H^{*}\left(S L_{q}\left(A_{k}\right) ; \mathbb{R}\right)
$$

where $G_{q}$ is the group of $\mathbb{R}$-points of an algebraic group defined over $\mathbb{Q}$ and whose $\mathbb{Q}$-points are $S L_{q}(k)$. But then $G_{q} \approx S L_{q}(\widehat{k}) \approx \prod_{v} S L_{q}\left(k_{v}\right)$, and $S L_{q}\left(A_{k}\right) \rightarrow G_{q}$ is the diagonal embedding, composed of all the embeddings $A_{k} \subset k \rightarrow k_{v}$.

$I_{G}$ is the algebra of $G$-invariant forms on the symmetric space of maximal compact subgroups of $G$. Then the main theorem is that $j$ becomes an isomorphism in degrees below a number that grows to infinity with $q$. (Borel's result is much more general, but all we need here is this special case.)

But it is a well known fact that $I_{G} \approx H_{\mathrm{LIE}}^{*}(\mathfrak{g} ; \mathfrak{k})$ where $K$ is a maximal compact subgroup of $G$, and the computation reduces to the Lie algebra homology computation already mentioned.

Consider the following diagram:

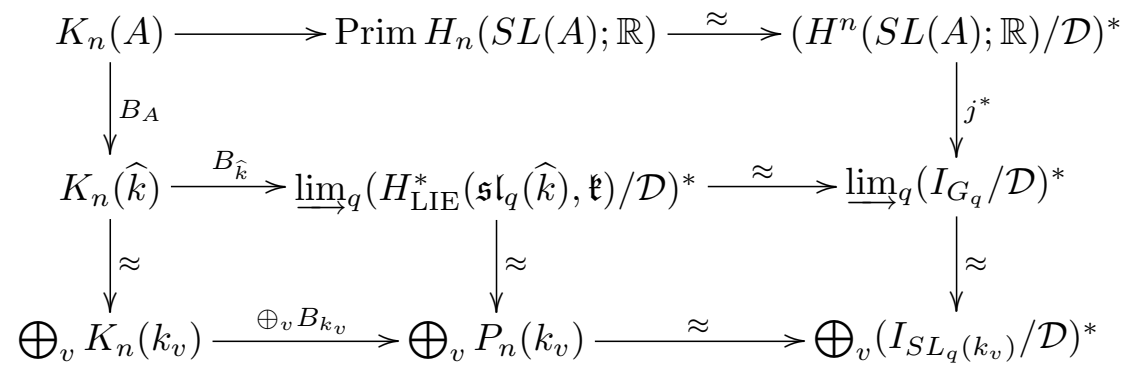

The lower half is obviously commutative, so we have to prove that the upper half commutes. 
The composition

$$
h: \Omega_{n}^{\mathrm{HS}}\left(B S L_{q}(A)\right) \rightarrow K_{n}(A) \rightarrow H_{n}(S L(A) ; \mathbb{R}) \rightarrow\left(H^{n}(S L(A) ; \mathbb{R})\right)^{*}
$$

takes $\left\{f: M \rightarrow B S L_{q}(A)\right\}$ to the class given by $c \mapsto c\left(f_{*}([M])\right)$, which factors through $\left(H^{n}\left(S L_{q}(A) ; \mathbb{R}\right)\right)^{*}$. It then suffices to prove that

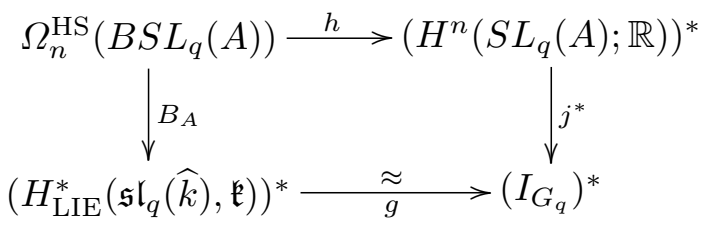

commutes for each $q$.

We need to make the two compositions very explicit. Let $G=G_{q}=$ $S L_{q}^{0}(\widehat{k}), K$ the maximal compact subgroup, and set $X=G / K . X$ is a contractible (diffeomorphic to a euclidean space) homogeneous $G$-space.

Then $I_{G}=\Omega^{*}(X)^{G} \approx \Lambda^{*}\left(T_{e} X\right) \approx \Lambda^{*}(\mathfrak{g})_{\mathfrak{k}}$, and the isomorphism $I_{G} \approx$ $H_{\text {LIE }}^{*}(\mathfrak{g} ; \mathfrak{k})$ is just the fact that differentials are trivial on both sides.

Let $P \rightarrow M$ be a principal $S L_{q}(A)$-bundle representing an element $x$ in $\Omega_{n}^{\mathrm{HS}}\left(B S L_{q}(A)\right)$. Let $\sigma: M \rightarrow P_{G} / K$ be the section associated with a $K$-reduction of the associated $G$-bundle, and $\omega$ as defined in Section 4. Then

$$
g B_{A}(x)(\phi)=\int_{M} \sigma^{*} \omega(\phi) .
$$

To compare this with the other composition, we need the following de Rham cohomology interpretation of $H^{n}\left(S L_{q}(A) ; \mathbb{R}\right)$ :

LeMma 5.5. $H^{n}\left(S L_{q}(A) ; \mathbb{R}\right) \approx H^{n}\left(\Omega^{*}(X)^{S L_{q}(A)}\right)$.

With this isomorphism, the map $j: I_{G} \rightarrow H^{*}\left(S L_{q}(A) ; \mathbb{R}\right)$ is induced by the inclusion $I_{G}=\Omega^{*}(X)^{G} \subset \Omega^{*}(X)^{S L_{q}(A)}$.

The statement in the lemma is well known, but we shall need the explicit isomorphism provided by the following

Proof. $S L_{q}(A)$ has a torsion free, normal subgroup $\Gamma$ of finite index. Then $\Gamma \backslash X$ is a manifold of the homotopy type of the classifying space $B \Gamma$. Since $\Omega^{*}(\Gamma \backslash X) \approx \Omega^{*}(X)^{\Gamma}$, we have $H^{*}(B \Gamma ; \mathbb{R}) \approx H^{*}\left(\Omega^{*}(X)^{\Gamma}\right)$ by the de Rham theorem.

Let $i^{*}: H^{*}\left(B S L_{q}(A) ; \mathbb{R}\right) \rightarrow H^{*}(B \Gamma ; \mathbb{R})$ be induced by the inclusion, and define $\operatorname{tr}: H^{*}(B \Gamma ; \mathbb{R}) \rightarrow H^{*}\left(B S L_{q}(A) ; \mathbb{R}\right)$ to be the transfer divided by the index $\left[S L_{q}(A): \Gamma\right]$ of $\Gamma$ in $S L_{q}(A)$, such that $\operatorname{tr} \circ i^{*}=$ id. Then $i^{*} \circ \operatorname{tr}$ is a projection on $H^{*}(B \Gamma ; \mathbb{R})$, with image $i^{*}\left(H^{*}\left(B S L_{q}(A) ; \mathbb{R}\right)\right) \approx$ $H^{*}\left(B S L_{q}(A) ; \mathbb{R}\right)$.

Both $i^{*}$ and tr have natural analogs as maps between $H^{n}\left(\Omega^{*}(X)^{S L_{q}(A)}\right)$ and $H^{n}\left(\Omega^{*}(X)^{\Gamma}\right): i^{*}$ is again induced by inclusion, and tr by averaging, 
using the action of $S L_{q}(A) / \Gamma$ on $\Gamma$. It is then easy to check that the de Rham isomorphism $H^{*}(B \Gamma ; \mathbb{R}) \approx H^{*}\left(\Omega^{*}(X)^{\Gamma}\right)$ commutes with the projections $i^{*} \circ$ tr. Hence it will induce an isomorphism between the two summands $H^{*}\left(B S L_{q}(A) ; \mathbb{R}\right)$ and $H^{*}\left(\Omega^{*}(X)^{S L_{q}(A)}\right)$.

Remark. It actually follows from Borel's computations that $i^{*}$ (hence also $\mathrm{tr}$ ) is an isomorphism in low degrees.

Let $\phi \in \Omega^{n}(X)^{S L_{q}(A)}$. We can describe the cochain $c(\phi)$ on $B S L_{q}(A)$ which $\phi$ determines by this isomorphism as follows:

Let $f: \triangle^{n} \rightarrow B S L_{q}(A)$ be a singular simplex, and let $\tilde{f}: \widetilde{\triangle}^{n} \rightarrow B \Gamma$ be the lifting of $f$ to the pullback of $B \Gamma \rightarrow B S L_{q}(A)$ over $f .\left(\widetilde{\triangle}^{n}\right.$ consists of $\left[S L_{q}(A): \Gamma\right]$ copies of $\triangle^{n}$.) Then

$$
c(\phi)(f)=\frac{1}{\left[S L_{q}(A): \Gamma\right]} \int_{\widetilde{\triangle}^{n}} \widetilde{f}^{*} \phi .
$$

Corollary 5.6. $h: \Omega_{n}^{\mathrm{HS}}\left(B S L_{q}(A)\right) \rightarrow\left(H^{n}\left(S L_{q}(A) ; \mathbb{R}\right)\right)^{*}$ is given by

$$
h(f)=\frac{1}{\left[S L_{q}(A): \Gamma\right]} \int_{\widetilde{M}_{\Gamma}} \tilde{f}^{*} \phi,
$$

where $f: M \rightarrow B S L_{q}(A)$ and $\widetilde{f}: \widetilde{M}_{\Gamma} \rightarrow B \Gamma$ is a lifting of $f$ to the pullback. $\left(\widetilde{M}_{\Gamma} \rightarrow M\right.$ is then an $\left[S L_{q}(A): \Gamma\right]$-fold covering space. $)$

To finish the proof of Theorem 5.2, we now need to give a description of the classifying map for a principal $S L_{q}(A)$-bundle over a manifold $M$. In fact, it follows from Corollary 5.6 that it suffices to do this for liftings $\widetilde{M}_{\Gamma} \rightarrow B \Gamma=\Gamma \backslash X$.

So, let $P_{A} \rightarrow M$ be an $S L_{q}(A)$-bundle, and let $P \rightarrow M$ be the associated flat $G$-bundle. Parallel transport with respect to the flat connection then determines a trivialization of the pullback $\varrho^{*} P$, where $\varrho: \widetilde{M} \rightarrow M$ is the universal covering space - hence also of $\varrho^{*} P / K$.

The section $\sigma: M \rightarrow P / K$ then pulls back to a section $\widetilde{\sigma}: \widetilde{M} \rightarrow \varrho^{*} P / K$, which we compose with the projection $\lambda$ to the fiber $G / K$ to give

$$
\gamma=\lambda \circ \sigma: \widetilde{M} \rightarrow X
$$

Note that there is a natural homomorphism $\pi_{1} M \rightarrow S L_{q}(A)$ (coming e.g. from parallel transport around loops in $P_{A} \rightarrow M$ ), and that $\gamma$ commutes with the actions of $\pi_{1} M$ and $S L_{q}(A)$ on $\widetilde{M}$ and $X$. Hence we get induced maps

$$
\gamma_{\Gamma}: \widetilde{M}_{\Gamma} \rightarrow \Gamma \backslash X
$$

for every $\Gamma \subset S L_{q}(A)$, where now $\widetilde{M}_{\Gamma}=\widetilde{M} / \varrho_{*}^{-1}(\Gamma)$. 
But we also have

$$
\gamma^{*}(\phi) \in \Omega^{*}(\widetilde{M})^{\pi_{1} M} \approx \Omega^{*}(M) \quad \text { if } \phi \in \Omega^{*}(X)^{S L_{q}(A)},
$$

so we can consider $\gamma^{*}$ as a homomorphism

$$
\gamma^{*}: \Omega^{*}(X)^{S L_{q}(A)} \rightarrow \Omega^{*}(M) .
$$

It follows that if $x \in \Omega_{n}^{\mathrm{HS}}\left(B S L_{q}(A)\right)$ is represented by $P_{A} \rightarrow M$, then $h(x)$ is given by

$$
h(x)(\phi)=\int_{M} \gamma^{*}(\phi) .
$$

(Hence we do not any longer refer to the finite index subgroup $\Gamma$.) It now only remains to observe that

$$
\sigma^{*} \circ \omega=\gamma^{*} \circ j^{*}=\widetilde{\sigma}^{*} \circ \lambda^{*}: \Lambda^{*}(\mathfrak{g})_{\mathfrak{k}} \rightarrow \Omega^{*}(M) .
$$

But this follows from the diagram

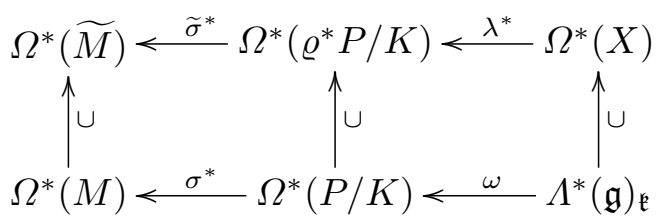

and the fact that $\omega$ is just an infinitesimal version of $\lambda$.

REMARK 5.7. Diagram (5.4) and the proof that it is commutative works for all $n$. Hence, for $n=3$, we find both that our invariant factors through $K_{3}$, and that we have the same interpretation of the Borel classes as in higher degrees. For $n=2$ or 4 we get zero in both cases, so the identification in Theorem 5.2 is actually valid for all $n>1$. In fact, using the interpretation in Remark 2.7 for $n=1$ and $G L_{q}$ instead of $S L_{q}$ (actually, it suffices to take $q=1)$, we get a map which we can identify with the usual embedding of $\left(A_{k}^{*} /\right.$ torsion $)$ as a codimension 1 lattice in $\mathbb{R}^{r_{1}+r_{2}}$.

6. The case of group rings. As another illustration, we take the example of group rings. For geometric applications, this is the most important example. We shall see that, properly interpreted, this case is very analogous to the case of rings of integers.

Let $\pi$ be a finite group. Then $\mathbb{R}[\pi]$ is a semisimple algebra, and

$$
\mathbb{R}[\pi] \approx \prod_{i} M_{d_{i}}\left(F_{i}\right), \quad \text { where } F_{i}=\mathbb{R}, \mathbb{C} \text { or } \mathbb{H} .
$$

The factors are matrix algebras corresponding to the irreducible, real representations of $\pi$. 
Then the homomorphism

$$
B_{\pi}: K_{n}(\mathbb{Z}[\pi]) \rightarrow K_{n}(\mathbb{R}[\pi]) \approx \prod_{i} K_{n}\left(F_{i}\right) \rightarrow \prod_{i} P_{i}\left(F_{i}\right)
$$

is in many ways similar to $B_{A}$ for rings of integers, and we have:

TheOREM 6.2. After tensoring with $\mathbb{R}, B_{\pi}$ becomes an isomorphism for $n>1$.

That is, we detect all of $K_{n}(\mathbb{Z}[\pi])$ except torsion by taking all the irreducible real representations of $\pi$ and using the idea of Section 4 !

The number of factors in the target of $B_{\pi}$ is equal to the number $r$ of irreducible real representations of $\pi$. By the definition of $P_{n}(F)$, this means that the rank is $r$ for $n \equiv 1(\bmod 4)($ and $>1)$, the number of real representations of complex type for $n \equiv 3(\bmod 4)$ and zero if $n$ is even.

The proof of Theorem 6.2 proceeds very much like the proof of Theorem 5.2, using the results of [6]. In fact, just as in Section 5 , we see that $B_{\pi}$ is equivalent to the dual of Borel's homomorphism

$$
j_{G L_{q}(\mathbb{Z}[\pi])}: I_{G L_{q}(\mathbb{R}[\pi])} / \mathcal{D} \rightarrow H^{*}\left(G L_{q}(\mathbb{Z}[\pi])\right) / \mathcal{D} .
$$

This is not quite good enough since $G L_{q}$ is not semisimple, but in [6] it is shown how one can define a semisimple replacement $S L_{q}(\mathbb{R}[\pi]) \supset$ $S L_{q}(\mathbb{Z}[\pi])$ - essentially by using the reduced norm for each factor of (6.1) and taking the kernel. Then Borel's result applies to give an isomorphism

$$
j_{S L(\mathbb{Z}[\pi])}: I_{S L(\mathbb{R}[\pi])} / \mathcal{D} \rightarrow H^{*}(S L(\mathbb{Z}[\pi])) / \mathcal{D} .
$$

For general groups we do not have a simple structure theorem such as (6.1), but $F$-representations $\varrho: \pi \rightarrow G L_{q}(F)$ still induce homomorphisms $\varrho: \mathbb{Z}[\pi] \rightarrow \mathbb{R}[\pi] \rightarrow M_{q}(F)$ and hence

$$
B_{\varrho}: K_{n}(\mathbb{Z}[\pi]) \rightarrow K_{n}\left(M_{q}(F)\right) \rightarrow K_{n}(F) \stackrel{B}{\rightarrow} P_{n}(F) .
$$

We can certainly detect elements this way, but in general $\operatorname{rank}\left(K_{n}(\mathbb{Z}[\pi])\right)$ may be nonzero also for even $n$. The simplest example is $\pi=\mathbb{Z}$, where e.g. $K_{6}(\mathbb{Z}[\pi]) \approx K_{6}(\mathbb{Z}) \oplus K_{5}(\mathbb{Z})$ has rank one.

\section{References}

[1] A. J. Berrick, Characterization of plus-constructive fibrations, Adv. Math. 48 (1983), 172-176.

[2] A. Borel, Stable real cohomology of arithmetic groups, Ann. Sci. École Norm. Sup. (4) 7 (1974), 235-272.

[3] - Cohomologie de $S L_{n}$ et valeurs de fonctions zêta aux points entiers, Ann. Scuola Norm. Sup. Pisa 4 (1977), 613-636. 
[4] J.-C. Hausmann, Homology sphere bordism and Quillen plus construction, in: Algebraic K-Theory (Evanston, 1976), Lecture Notes in Math. 551, Springer, 1976, $170-181$.

[5] J.-C. Hausmann and P. Vogel, The plus construction and lifting maps from manifolds, in: Proc. Sympos. Pure Math. 32, Amer. Math. Soc., 1978, 67-76.

[6] B. Jahren, On the rational $K$-theory of group rings of finite groups, preprint, Oslo, 1993.

[7] J. D. S. Jones and B. W. Westbury, Algebraic K-theory, homology spheres, and the $\eta$-invariant, Warwick preprint 4/1993.

[8] F. W. Kamber and Ph. Tondeur, Foliated Bundles and Characteristic Classes, Lecture Notes in Math. 493, Springer, 1975.

[9] M. Karoubi, Homologie cyclique et K-théorie, Astérisque 149 (1987).

[10] M. A. Kervaire, Smooth homology spheres and their fundamental groups, Trans. Amer. Math. Soc. 144 (1969), 67-72.

[11] S. Lichtenbaum, Values of zeta functions, étale cohomology, and algebraic K-theory, in: Algebraic K-Theory II, Lecture Notes in Math. 342, Springer, 1973, 489-501.

Department of Mathematics

University of Oslo

P.O. Box 1053 Blindern

0316 Oslo, Norway

E-mail: bjoernj@math.uio.no 